\title{
Statistical Methods in Organ Failure and Transplantation
}

\author{
Douglas E. Schaubel $^{1}$
}

Published online: 27 November 2017

(C) International Chinese Statistical Association 2017

The special issue contains ten stimulating articles which feature the development of novel statistical methodology applied to important issues in organ failure and transplantation. Several of the articles feature survival analysis, which has a long history in the organ failure setting, dating back to (at least) the seminal analysis of the Stanford heart transplant data by Crowley and Hu [1]. Fields such as end-stage renal disease (ESRD) and end-stage liver disease (ESLD) are among the many areas of medicine which have been particularly receptive to (and, as a result, heavily influenced by) innovative statistical techniques.

This issue spans several domains with respect to statistical methodology and areas of application, including instrumental variables, competing risks, recurrent events, gap times, landmark analysis, dynamic prediction of outcomes, joint modeling of longitudinal and time-to-event data, and variable selection. Methodologists and practitioners whose area of application is outside the organ failure setting will find plenty of stimulating material in this issue.

Lehmann et al. [2] propose a weighting approach for instrumental variable (IV) analysis, designed to make the IV more robust to the critical assumption of random assignment. Using data obtained from the United States Renal Data System (USRDS), the proposed methods are used to compare mortality on hemodialysis versus peritoneal dialysis, a long-standing yet unresolved issue in ESRD treatment. The increasingly important topic of kidney paired donation (KPD) is studied by Wang et al. [3], who develop a novel multiple-look-ahead decision strategy to select transplant chains.

Huang et al. [4] consider recurrent event data and, in particular, the setting where each recurrent event is subject to competing risks. Subdistribution hazard regres-

Douglas E. Schaubel deschau@umich.edu

1 Department of Biostatistics, University of Michigan, 1415 Washington Heights, Ann Arbor, MI 48109-2029, USA 
sion methods are proposed and applied to analyze shunt thrombosis events among Taiwanese dialysis patients. Competing risks are also considered by Yang et al. [5] who jointly model repeated measures and competing failure events. The methods are applied to the Chronic Renal Insufficiency Cohort (CRIC) study to determine risk factors for progression from chronic kidney disease (CKD) to ESRD.

A general framework for landmark analysis is developed by $\mathrm{Li}$ et al. [6]. The methods are used to dynamically project ESRD and death among CKD patients. Putter and van Houwelingen [7] establish connections between landmark analysis and timedependent Cox regression, both theoretically and empirically.

Penalized variable selection techniques are proposed for the competing risks setting in the article by Fu et al. [8]. The methods are applied to data clustered by center and are used to determine risk factors for adverse post-transplant outcomes. We return to variable selection in the article by Zhou et al. [9]. The authors apply a LASSO machine learning algorithm in the context of Cox regression, with the goal being to identify proteins associated with graft failure among kidney transplant patients.

Nguyen and Gillen [10] propose a survival-tree approach to identify group-specific censoring patterns in order to estimate the average hazard ratio under a misspecified proportional hazards model. The methods are motivated by estimating the effect of vascular access type with respect to time until access revision among hemodialysis patients. Methods for comparing gap times are proposed by Shu and Schaubel [11]. The authors develop techniques for estimating the gap time hazard ratio, with the application being to compare primary versus repeat liver transplant survival.

I would like to thank the authors of the exciting articles appearing in this issue for their interesting and important contributions. On behalf of the authors, I thank MeiCheng Wang, Co-Editor-in-Chief of Statistics in Biosciences, for her encouragement to assemble a special issue dedicated to transplantation. I also wish to thank Mei-Cheng for giving me the opportunity and honor to serve as Guest Editor for this issue.

\section{References}

1. Crowley J, Hu M (1977) Covariance analysis of heart transplant survival data. J Am Stat Assoc 72:2736

2. Lehmann D, Li Y, Saran R, Li Y (2018) Strengthening instrumental variables through weighting. Stat Biosci, 1-19

3. Wang W, Bray M, Song PXK, Kalbfleisch JD (2018) A look-ahead strategy for non-directed donors in kidney paired-donation. Stat Biosci, 1-17

4. Huang C-H, Li B, Chen C-M, Wang W, Chen Y-H (2018) Subdistribution regression for recurrent events under competing risks: with application to shunt thrombosis study in dialysis patients. Stat Biosci, 1-8

5. Yang W, Xie D, Pan Q, Feldman HI, Guo W (2016) Joint modeling of repeated measures and competing failure events in a study of chronic kidney disease. Stat Biosci, 1-21

6. Li L, Luo S, Hu D, Greene T (2018) Dynamic prediction of renal failure using longitudinal biomarkers in a cohort study of chronic kidney disease. Stat Biosci, 1-22

7. Putter H, van Houwelingen HC (2018) Understanding landmarking and its relation with time-dependent Cox regression. Stat Biosci, 1-5

8. Fu Z, Ma S, Lin H, Parikh CR, Zhou B (2018) Penalized variable selection for multi-center competing risks data. Stat Biosci, 1-27

9. Zhou L, Tang L, Song AT, Cibrik DM, Song PXK (2018) A LASSO method to identify protein signature predicting post-transplant renal graft survival. Stat Biosci, 1-22 
10. Nguyen VQ, Gillen DL (2018) Censoring-robust estimation in observational survival studies: assessing the relative effectiveness of vascular access type on patency among end-stage renal disease patients. Stat Biosci, 1-25

11. Shu X, Schaubel DE (2018) Methods for contrasting gap time hazard functions: application to repeat liver transplantation. Stat Biosci, 1-9 